\title{
A Multi-Resolution Design Methodology Based on Discrete Models
}

\author{
Manuel Ladron de Guevara ${ }^{1}$, Luis Borunda ${ }^{2}$, Ramesh Krishnamurti ${ }^{3}$ \\ ${ }^{1}$ Carnegie Mellon University \\ manuelr@andrew.cmu.edu \\ ${ }^{2}$ Universidad Politecnica de Madrid \\ lborunda.eco@etsav.cat \\ ${ }^{3}$ Carnegie Mellon University \\ ramesh@andrew.cmu.edu
}

\begin{abstract}
The use of programming languages in design opens up unexplored and previously unworkable territories, mainly, in conventional architectural practice. In the 1990s, languages of continuity, smoothness and seamlessness dominated the architectural inquiry with the $\mathrm{CNC}$ milling machine as its manufacturing tool. Today's computational design and fabrication technology look at languages of synthesis of fragments or particles, with the 3D printer as its fabrication archetype. Fundamental to this idea is the concept of resolutionthe amount of information stored at any localized area. Construction of a shape is then based on multiple areas of resolution. This paper explores a novel design methodology that takes this concept of resolutions on discrete elements as a design driver for architectural practice. This research has been tested primarily through additive manufacturing techniques.
\end{abstract}

Keywords: Multi-Resolution Design Methodology; Discrete-Based

Computational Design; Resolutions; Additive Manufacturing.

\section{$1 \quad$ Introduction}

Resolution is defined as the amount of data in a given area. Its application in architecture is relatively new, since conventional design processes do not operate on such raw data. Instead, architects manipulate geometries. In other fields, for example, imaging, the term resolution reflects the detail an image holds. Digital images measure resolution by its number of pixels each containing a masked color or grayscale value as data. Historically, resolution has been an issue in communication systems, as the transmission of images entails data conversion from the transmitting station to the receiving station; inventors Sharp and Thompson designed methods for augmenting resolution [1]. 
In architecture, resolution has been explored by architects Dillenburger and Hansmeyer who define it as the number of voxels per volume, looking at the maximum resolution at which an additive manufacturing (AM) method can fabricate [2]. In this research, however, resolution is the quantity of information at a localized area of a discretized 3-dimentional shape. It is important to note that a shape designed by a conventional design model cannot be measured by its resolution, for instance, when geometry is manipulated using a modeling software. A resolution-based methodology is necessarily driven by manipulation of data through the use of programming tools.

Today's computational technologies offer the possibility to directly design in 3dimensions without recourse to encoding and decoding shapes via 2-dimensional drawings. Furthermore, programming languages provide a means of constructing shapes through raw data without geometrical manipulation. Prior to the use of algorithms, the design process was purely intuitive, and designers normally made decisions based on what they perceived from visual feedback [3].

Technological advancement often comes with a period of exploration in which architects learn how to implement them [4]. In the late 1980s, with the introduction of CAD software, manual drafting was substituted by digital drafting; during the 1990s, software developed for industries such as film- Autodesk's 3D Studio, or aeronauticsDassault Systemès' CATIA, were appropriated. Nonetheless, the essential paradigm remained the same: manipulation of geometry and modification based on visual feedback. On the fabrication side, the combination of CAD/CAM technologies created a seamless connection between design and manufacturing, which gave architects the possibility to re-gain their lost status as master builders [5].

Parametric tools, such as Grasshopper and Dynamo, have generalized visual scripting, augmenting the design process by the insertion of procedural logic and data management, facilitating contemporary avant-garde architectural styles such as, parametricism [6]. However, such tools neither, necessarily, address design in terms of discreteness nor resolution.

Discrete methods are normally used by analytical frameworks, for instance, finite element method (FEM), a numerical technique for solving problems such as structural analysis, heat transfer or fluid flow. An object is discretized into smaller units that are studied separately, yet as part of an integrative process. Karamba 3D by Clemens Preisinger or STAAD.Pro by Bentley, are examples of force distribution solvers. While these analyses can be integrated in the design process, there is no such generalized discrete design methodology.

This paper provides a step towards a new methodology of design, based on multiresolution adjustments, using a discretization technique, which crafts raw data, without manipulating geometry. Resolution measured by the quantity of data in a given area is natural to this method. A localized area comprises a cluster of neighboring fragments, with information stored in a set of dictionary data structures, containing numerical and textual values, such as coordinates, material information, location, context awareness and Boolean values. Shape is understood as multiple dictionaries of information, which define its resolutions levels. In essence, this data construes discrete geometrical units informed by the fabrication process. 
Additive fabrication technology needs a certain amount of data and machine time, does not involve reusable cast, mold or stamp, and does not require any voxel-generated volume to be identical to any other, regardless of scale or size. The marginal cost of a voxel is always the same, no matter how many we print. In this way, 3D printing brings the logic of customization from the macro to the micro scale of production of physical matter, and at previously unimagined levels of complexity and granularity [7]. Utilization of additive manufacturing methods is clearly ideal when designing with different resolutions.

\section{State of the Art}

\subsection{Resolution}

The notion of resolution as a driver in design fields is relatively new-it is linked to a recent computational revolution in which brand new science begets a new way of thinking, and to the advent of additive fabrication such as 3D printing [4]. The following designers include the word "resolution" in their descriptions. Voxel Chair and Curvoxels by Garcia and Retsin, use a discrete approach specifically to directly design for a 3D Spatial printing fabrication [8], [9]. However, their design approach is not driven by concepts of resolution and is rather a medium for a specific fabrication method. The Computational Chair Design Studies by Phillipe Morel of EZCT Architecture \& Design were among the earliest demonstrations of discrete approaches. Interestingly, a Finite Element Analysis (FEA) framework is used as a design tool. Digital Grotesque, by Dillenburger and Hansmeyer, is the first work to tackle resolution as a driver in design and fabrication. Their work is a manifestation of exuberance and a celebration of computational power [2]. While resolution is their main motive, their project looks at the highest resolution possible, rather than taking resolution as a platform for multiple inquiry.

Recently, Alisa Andrasek showcased the Croatian National Pavilion, Cloud Pergola, at the Venice Biennale [10]. A large-scale robotic spatial printing workpiece is designed using algorithms for multi-agent systems (MAS). Her algorithm computes active discrete elements whose behavior is determined by a collection of rules. Although the resolution is the same across the piece, we can see the potential in designing through discrete approaches: this strategy yields a high performance at structural, material wastage and aesthetic capacities.

In other fields, common use of discrete methods includes volumetric imaging in medicine, representation of terrain in games and simulations, and granular flows and rock mechanics in engineering. A crucial stage in volume graphics is the synthesis of voxel-represented objects. This stage, called voxelization, is concerned with converting geometric objects from their continuous geometric representation into a set of voxels that approximates the object [11]. 


\subsection{Fabrication}

Additive manufacturing enables the fabrication of complex physical designs. One substantial research development specific to AM is the use of spatial deposition techniques[12], [13] capable of continuously extruding cellular lattice members in a spatial organization with minimal waste [14], [15]. Foams and spatial lattices provide multifunctional lightweight structures, high strength and relatively low mass [16], [17] and robotic AM [18] the precision required to manufacture. In order to manufacture complex geometries through a continuous deposition method, fabrication and computational methods that serialize the deposition process into a concatenation of units have been developed [9].

Research efforts focus on adapting resolution in the discretization process while maintaining the index concatenation required for the geometrically correct continuous deposition (Fig. 1). We can build upon advances in AM application to other disciplines such as bone reconstruction of graded porosity [19]-[21], in particular, to study bones at an architectural level in order to establish hierarchically and topologically different design principles [22], [23]. This research provides a spatial organization system of indexable units with differentiated performance, boundary and neighboring conditions of manufacture through continuous deposition maintaining geometrical continuity.

With resolution based design, such strategies may be translated into functional largescale architectural components with potential for complex design of engineered anisotropy [24], [25] feasible through spatial AM.

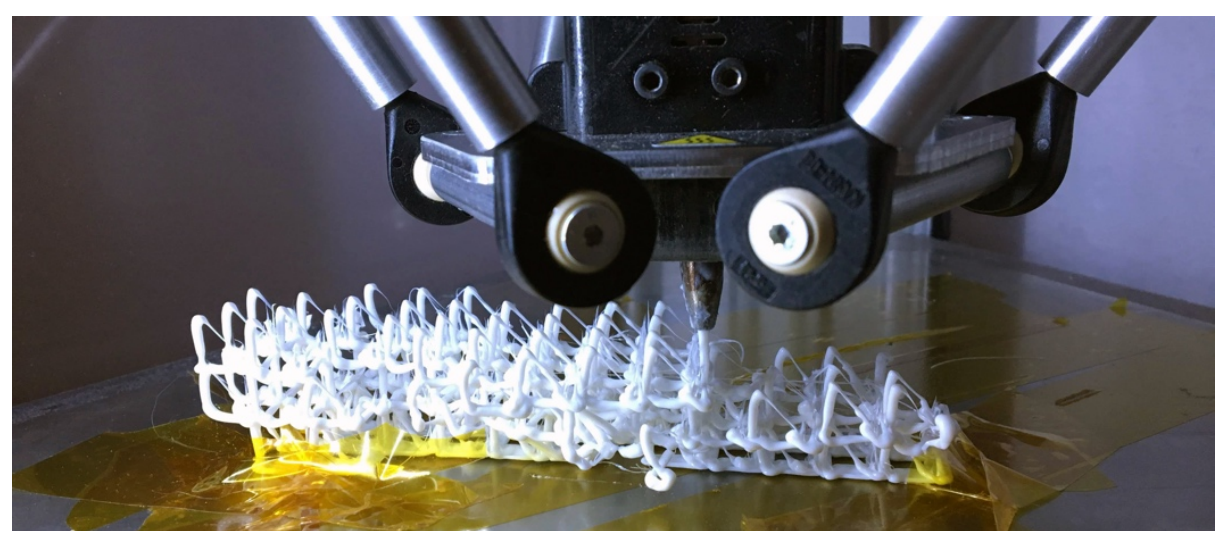

Fig. 1. 3D Spatial printing technique with a commercial delta 3D printer.

\section{$3 \quad$ Method}

\subsection{Overview}

The method proposed here examines the potential of designing an object using a discrete elements approach in order to configure its morphology to specific conditions through multi-resolution adjustments. The balance between higher and lower 
resolutions across the object corresponds to different design criteria such as aesthetic values, structural behavior, density, and material properties. Higher resolutions correspond to interesting areas, and lower resolutions target less interesting areas. That is, the algorithm behaves differently according to the degree of resolution, where the subdivision level quantifies the interesting areas and the type of information that qualifies them.

The number of areas of interest follow a design criterion, which depends on user demand, thus, the algorithm solution can be expanded, more or less, upon this. For this particular research, the algorithm considers variable density, opacity levels, and printable in-budget implementations.

The algorithm is divided into three main parts: shape analysis, grid structure, and discrete elements design. Understanding the fabrication end is imperative in this approach, since the affordances and limitations of material and tool define the design possibilities.

This research provides a step towards a design methodology based on multiresolution adjustments. For simplicity, we show the potential of the method through the study of an ulna bone (Fig. 2) both as a design example, and as a potential application of this design and fabrication approach to other fields. Current research focuses on expanding the algorithm to a larger scale for architectural practice.

The algorithms are written in Python, and for visualization purposes, Grasshopper is used to transform numerical data into geometrical values, using the Rhino Common library.

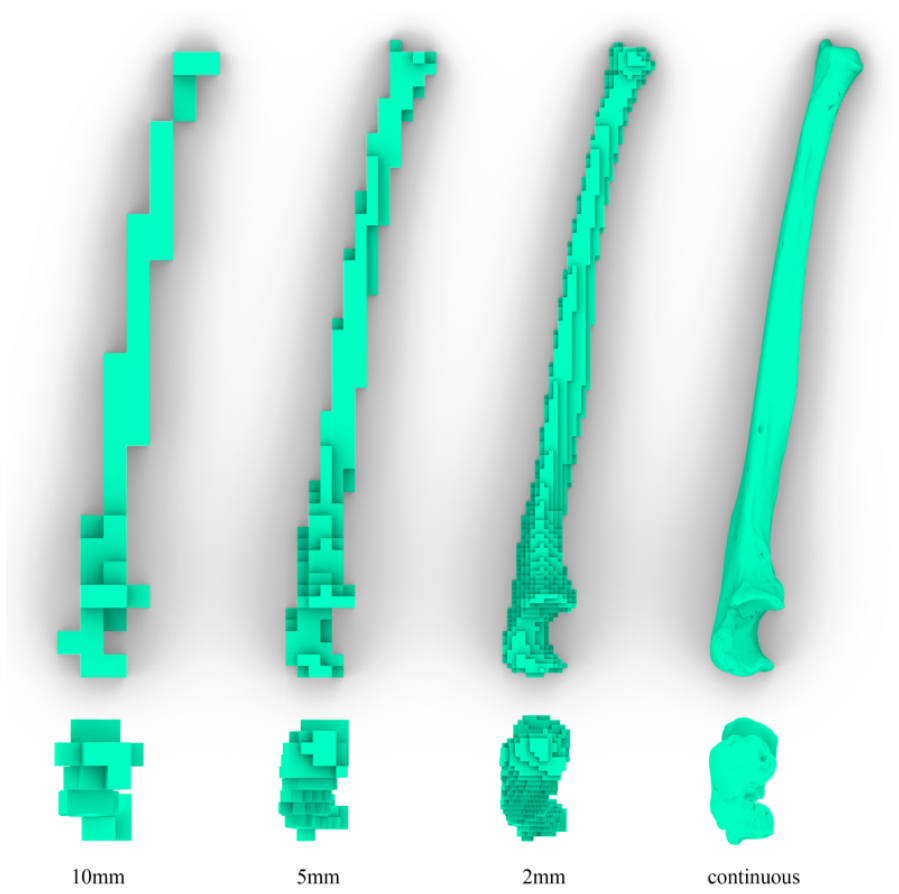

Fig. 2. Discrete and continuous models of the ulna bone 


\subsection{Shape Analysis and Discretization Structure}

The design process begins by taking the main geometry to be discretized as a closed mesh, surface or poly-surface. Its boundaries are calculated, and the mesh repositioned at the origin. A 3-dimentional multi-sized grid is constructed and automatically adjusted to the size of the initial volume, based on the number of rows, columns and floors, with values corresponding to the respective boundary dimensions divided by a default resolution size. This resolution value, $R$, specifies the size of a voxel $R \times R \times R$ (Fig. 3). The grid structure is used to specify the voxels that lie inside or outside the initial volume through an octree data structure [26] of true/false values. Computationally, the grid defines an object class, containing dictionaries of data of the interesting area, subdivision levels, and geometry and material properties.

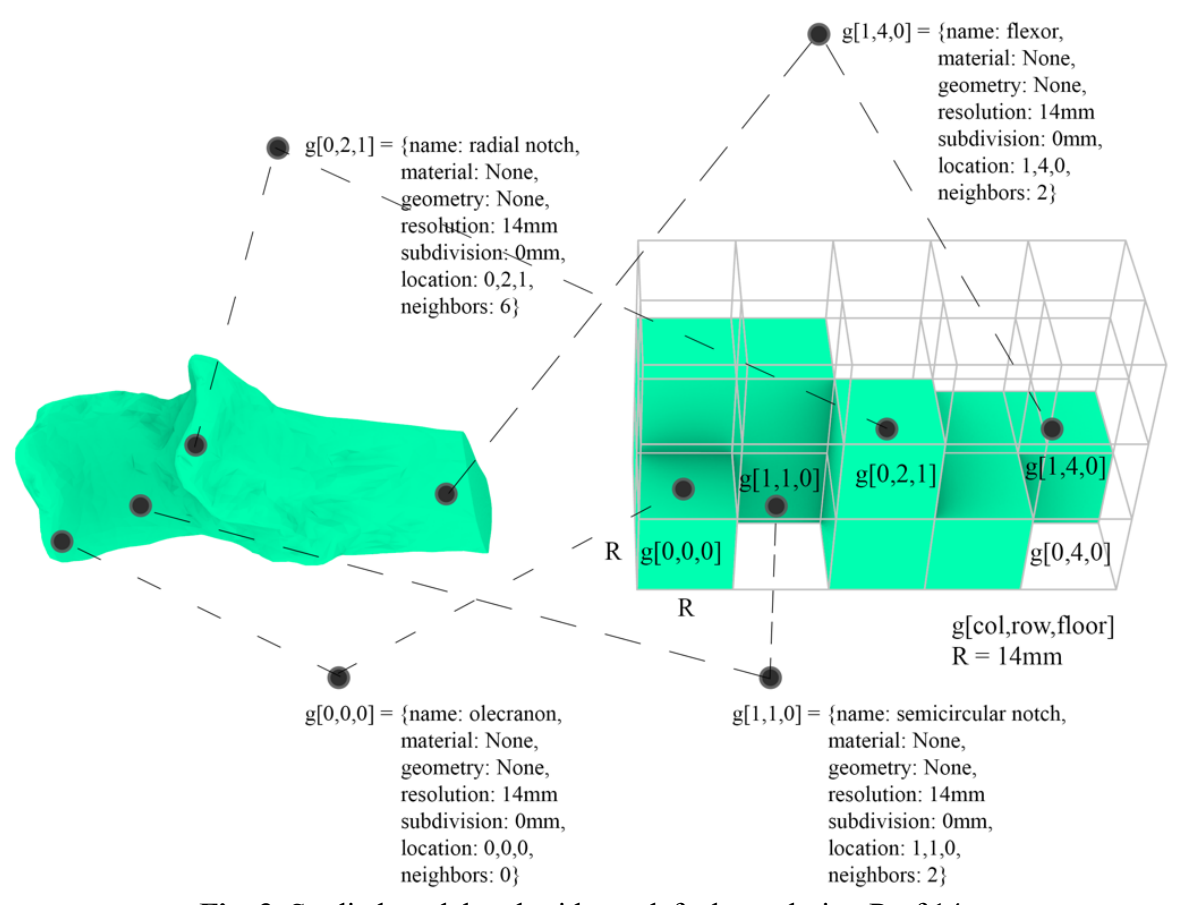

Fig. 3. Studied model and grid at a default resolution R of $14 \mathrm{~mm}$

\subsection{Arxel}

An Arxel is an informed digital architectural unit. It is a voxel with an overlapping agenda of material properties, economy, geometry and performative data such as structural behavior, opacity and density variations. Arxels are understood in dependency with their surrounding arxels. As a data unit an arxel is an information container, rather than a geometrical element, and it is structured as a set of dictionaries. 
Its boundary is defined by eight coordinates corresponding to its vertices. The boundary, however, is only for visualization purposes; it does not possess any geometrical value.

\subsection{Multi-Resolution}

The resolutions of the main volume are constructed by the scrutiny of interesting areas. A volume can have one or more different resolutions. A default resolution is defined at the beginning of the process, normally informed by the fabrication tool, and new resolutions are given by the said interesting areas. In our implementation these are selected directly in the Rhino view-space and linked to Grasshopper. The subdivision level corresponding to these areas responds to a design criterion and limitations on the fabrication, where the algorithm is able to compute different degrees of resolution altogether (Fig. 4). Ray casting is used to retrieve the Boolean value at each arxel, yielding true, if its centroid falls inside the interesting areas, and false, otherwise. This information is passed in the form of a dictionary, which is then read by a function that subdivides the arxels based on the subdivision level. Level $=1$ subdivides the arxel into 8 smaller units, level 2 subdivides it into 64 units, and so on. An algorithm similar to an octree approach is developed to form a multi-resolution grid. The algorithm restricts each arxel with a false value to level 0 , the default level of resolution. In order to bridge between a conventional IDE and the Rhino-3D environment and geometry created in GH-Python, the grid information is exported as a Javascript json file.

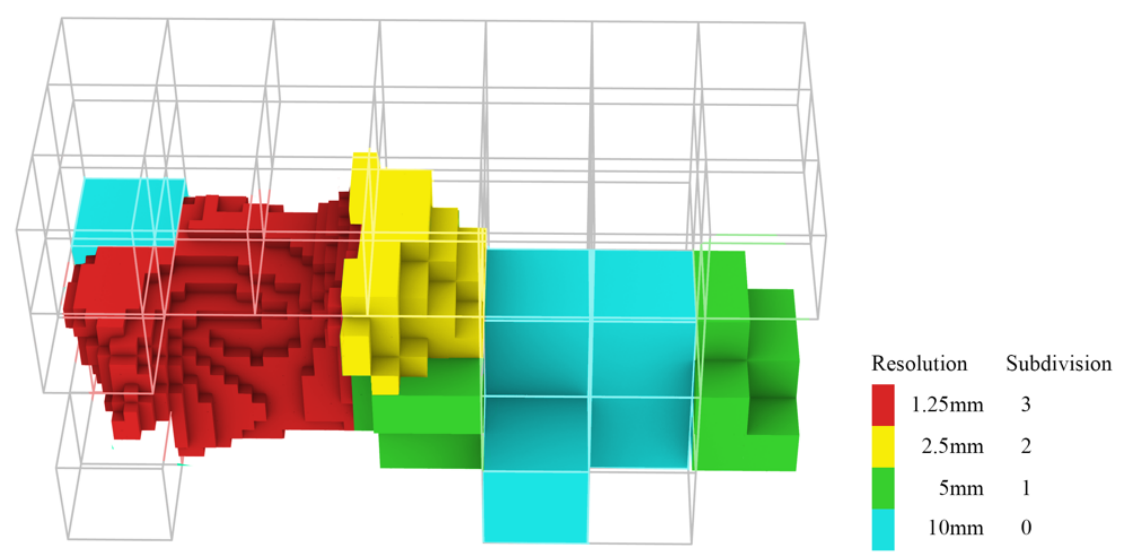

Fig. 4. Resolutions levels

\subsection{Design Discreteness}

This section has two parts. The first describes a general approach to design discreteness without considering limitations of fabrication; the second implements specific features such as material budget responsiveness and consideration of 
constraints on the 3D spatial printing technique for manufacturing purposes, such as printing order and collision checks.

\subsubsection{Basic Configuration}

This process takes, as input, information relating to the resolutions of the object from the previously exported data file, to start the design. This is informed by the fabrication side, and natural to the process is the creation of a linear language where the geometry is directly used as toolpath [9], [27]. However, we insert different XYZ-coordinates that are connected with lines only for purposes of visualization. The creation and manipulation of geometry is restricted to the management of numerical values (1). For instance, a rotation of an arxel through angle $\alpha$ is given by:

$$
\begin{aligned}
& x^{\prime}=x \cos \alpha-y \sin \alpha \\
& y^{\prime}=y \sin \alpha+y \cos \alpha
\end{aligned}
$$

No direct manipulation of geometrical models is made. A set of six numerical matrix configurations is designed, increasing in shape complexity to ensure disparity between the options at shown in Fig. 5. For instance, an area within the given volume that may require a higher structural reinforcement could be achieved by placing denser geometries to the detriment of simpler ones. Similarly, areas with higher degrees of opacity might be better determined by denser geometries. Each geometrical configuration is placed within the boundary of an arxel, selected upon design specifications. In order to increase options for design and fabrication, four $90^{\circ}$ rotations along the $\mathrm{Z}$ axis are also added to the set, augmenting each initial grammar by four, having a set of 24 shapes.
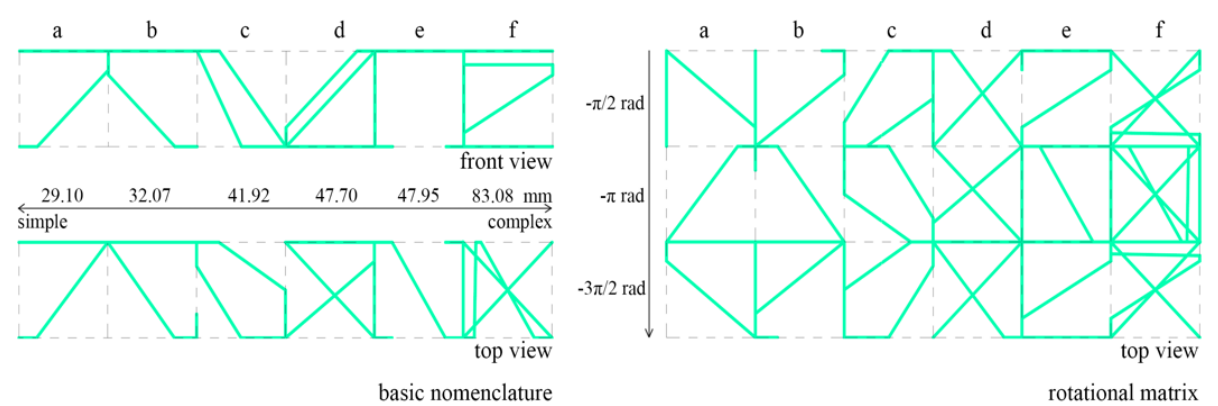

basic nomenclature

rotational matrix

Fig. 5. Matrix configurations

\subsubsection{Basic Configuration}

Fabrication is based on 3D spatial printing techniques: we create a design language based on linear segments, constrained by material and tool properties. For instance, a delta type 3D printer, which extrudes $1.75 \mathrm{~mm}$ filaments of polylactic acid (PLA) or 
acrylonitrile butadiene styrene (ABS), will constrain certain design decisions. The nozzle of the printer defines the angle between the linear segments of a polyline and the height of an arxel.

The printing order informs the position of the numerical configurations at each arxel to avoid collisions with already printed geometries. The printing order follows the logical positioning of the arxels within the grid, in the order of columns (Y axis), rows ( $\mathrm{X}$ axis) and floors ( $\mathrm{Z}$ axis). That is the algorithm starts positioning the first numerical matrix at the $0^{\text {th }}$ column, $0^{\text {th }}$ row and $0^{\text {th }}$ floor indices, the next matrix is placed at the $1^{\text {st }}$ column, $0^{\text {th }}$ row, and $0^{\text {th }}$ floor. At this point it is necessary to check whether the chosen matrix may collide with an already printed neighboring arxels. For instance, at the position $\mathrm{col}=1$, row $=0$, floor $=0$, only the arxel at position (col- 1 , row) needs to be checked. At the position, say, col $=3$, row $=5$, floor $=0$, the list of arxels $[($ col-1,row- 1$)$, (col-1,row), (col+1,row), (col,row-1)] should be checked. A backtracking algorithm is implemented to find a valid solution following the pseudo-code below:

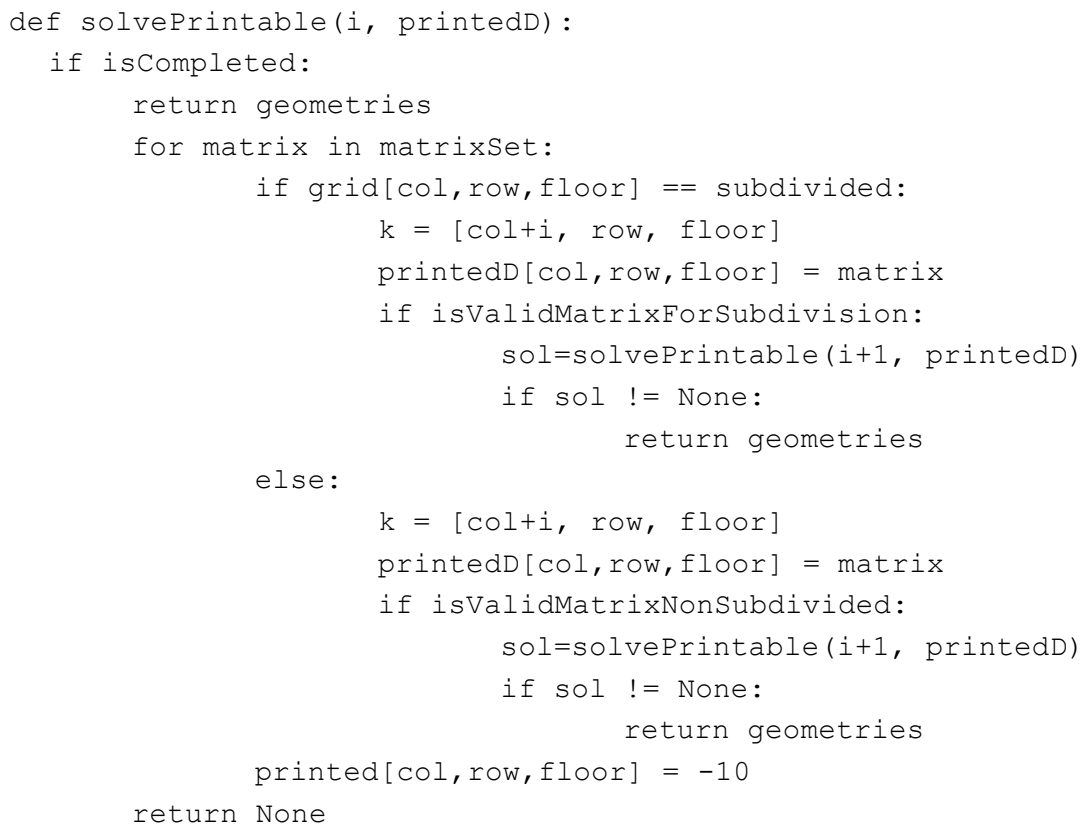

To check whether an arxel might collide with previously printed arxels, a comparison is made between each of its coordinates and the already printed coordinates, such that $\mathrm{zP}>\mathrm{z},-\mathrm{xP}<\mathrm{x}<\mathrm{xP}$ and $-\mathrm{yP}<\mathrm{y}<\mathrm{yP}$, being $\mathrm{xP}, \mathrm{yP}, \mathrm{zP}$ already printed coordinates. The domains $\{-\mathrm{xP},+\mathrm{xP}\}$ and $\{-\mathrm{yP},+\mathrm{yP}\}$ correspond to the nozzle dimensions. The complexity of the algorithm increases at the subdivided voxels, since these arxels are formed by matrixes of matrixes equivalent to the level of resolution.

Similar to the backtracking strategy explained above, an algorithm that finds a valid solution responding to material length limit is computed. To adjust the overall piece 
within a material constraint, calculation of the distance of the coordinates at each voxel is required in order to know the total printed length of the piece. If the total length is more than the given material budget value, the algorithm selects pseudo-randomly nonsubdivided arxels, since these correspond to less interesting areas. Their matrix is changed and tested procedurally, passing tests if the overall length is less than the previous state and still above the material budget. If so, another arxel is selected, changed and tested until finding a solution that fits in the budget. If no solution is found, earlier design decisions such as the size or quantity of interesting areas, or the base resolution should be modified.

\section{Case Study}

An ulna bone is used as the case study since its complex morphology requires different levels of resolutions. Furthermore, applying our design approach at a human scale first will inform bigger and more complex architectures. We apply a higher degree of resolution to the ulna's radial and semicircular notch through the definition of a localized sphere, and also, we reinforce the ulna's coronoid process via definition of a spline (Fig. 6). We maintain lower resolutions in the rest of the bone.

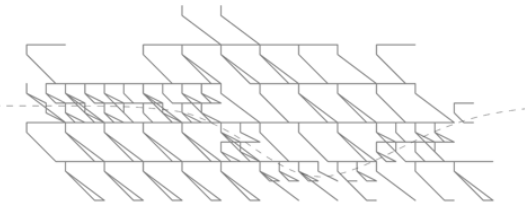

front view

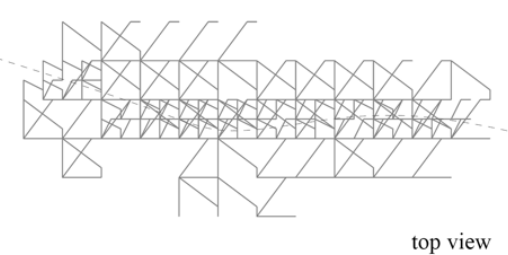

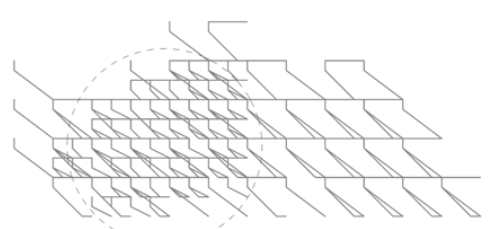

front view

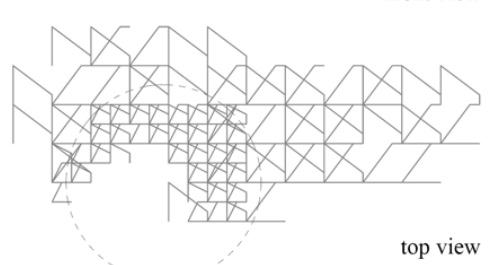

Fig. 6. Selection by spline (left) and by volume (right)

The case study begins with an analysis of the ulna, extracting its dimensions and decreasing the scale by two thirds for fabrication in a conventional 3D delta printer. Initial tests with different defaulted resolution are made to assess the degree of information that satisfies the purposes of this research. For the initial analysis, we use the arxels' boundary representation, that is, equilateral cuboids, for visualization purposes. Upon determining the interesting areas of the ulna, we compute the levels of subdivision based on the amount of information at the notch and at the internal stress line as shown in Fig. 6. The design process continues by automated selection of the 
type of geometry that best suits the specific location at the bone. That is, since the grid stores information on the piece such as the number of components or parts, areas of interest or structural analysis, we define denser areas where a higher structural capacity is required, and the algorithm selects those grammars within the set that fit the needs.

Once the selection of the resolutions and type of geometry of the discrete elements that form the bone satisfies the design conditions, the backtracking algorithm for fabrication is enacted, where a fabrication solution is found without altering the situated conditions across the piece. For simplicity, no multi-material is implemented since we use a conventional nozzle in the 3D printer. Instead, PLA is used for early prototyping (Fig. 7).

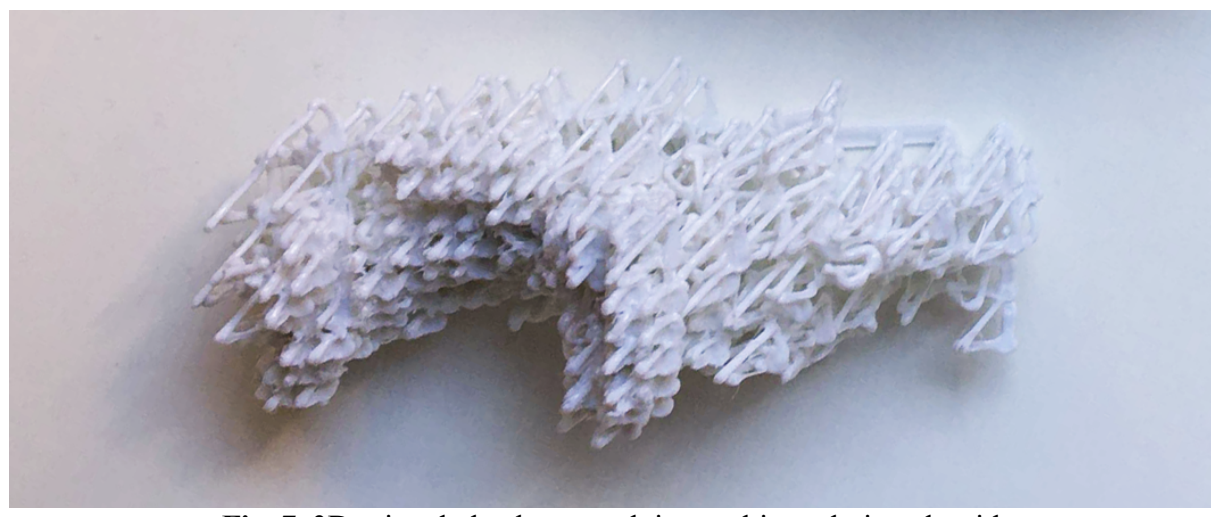

Fig. 7. 3D printed ulna bone applying multi-resolution algorithm

\section{$5 \quad$ Results and Discussions}

This paper contributes to a design methodology based on multi-resolution adjustments as areas of opportunity. This is only now feasible as the design methodology represents a change in design thinking spurred by a computational revolution and the advent of $3 \mathrm{D}$ printers. It is now when we can start talking about resolution in architecture.

The design method presents numerous limitations that should be overcome with more research in both the computational part and physical tool development. This research uses recursion as a main strategy for subdivision; this is computationally expensive and relatively complex. On the other hand, subdivision follows a recursive logic that might be quicker to apply than other alternatives. Data structures such as dictionaries or sets are used, as their hashable nature optimizes the operation time. Also, designing within a frame of spatial printing presents even more constraints. The fidelity between the digital model and the physical model fabricated in a $3 \mathrm{D}$ delta printer differs around a 17\%. The segment-like nature of the geometry also limits the design scope. Combined strategies between spatial printing and conventional 3D printing is under research by the authors. 
The ulna bone was chosen as the design study, since the degree of complexity of a bone is convenient to assess the potential benefits and limitations of this design approach. Moreover, understanding it at a human scale informs possible design considerations at an architectural scale. The authors of this paper acknowledge that this is only a small step towards a larger design process. However, this design approach can be understood at a higher level, by considering new computational and fabrication tools available to explore alternative design methods. We have used a multi-resolution approach that is limited to different geometrical and scale outputs that define structural behavior and different density levels. However, this approach can be expanded in multimateriality and multi-fabrication techniques, and also the exploration of novel aesthetic meanings. Similar to the shift of the meaning of the value of the ornament in architecture [7], new aesthetic values might arise as the result of design alternatives.

Although this paper attempts to generalize a discrete approach, we understand that a relatively high level of computation is needed in order to follow this design process. Also, a sophisticated understanding of the material and the machine is required if the design seeks to leave the digital environment. The physical pieces presented in this paper (Fig. 8) require some level of knowledge about hacking commercial 3D printers. The design space is conducive to creative exploration, and the understanding of resolution through other fabrication techniques remains open.

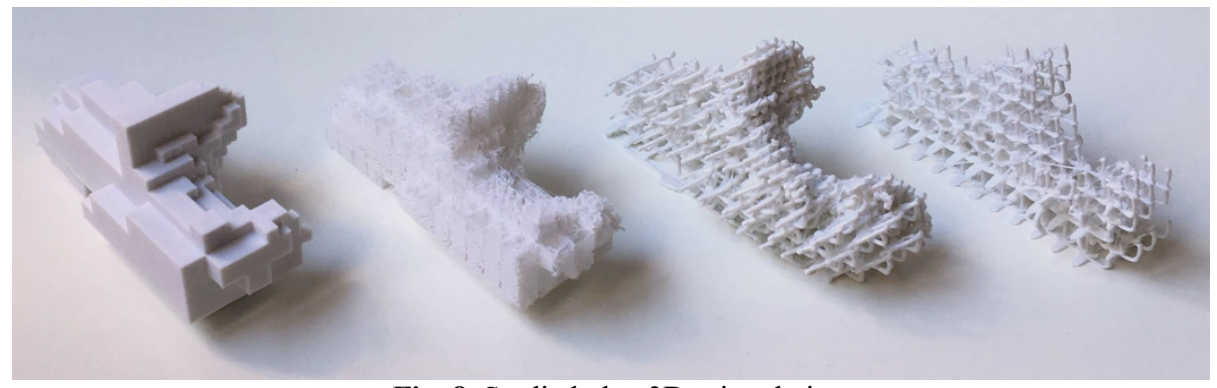

Fig. 8. Studied ulna 3D printed pieces

Acknowledgements. The authors would like to thank the following organizations that funded this research; The Frank Ratchye Fund for Art @ the Frontier (FRFAF),Consejo Social de la Universidad Politécnica de Madrid and the National Council of Science and Technology of Mexico.

\section{References}

1. J. V Sharp and D. R. Thompson, "Method and apparatus for increasing image resolution." Google Patents, (1971)

2. B. Dillenburger and M. Hansmeyer, The Resolution of Architecture in the Digital Age, 369 (2013) 
3. R. Oxman, "Educating the designerly thinker," Des. Stud., 20, no. 2, pp. 105-122 (1999)

4. M. Carpo, The second digital turn : design beyond intelligence. MIT Press, (2017)

5. D. Cardoso, "Builders of the Vision," p. 208 (2012)

6. P. Schumacher, "Parametricism: A new global style for architecture and urban design," Archit. Des., 79, no. 4, 14-23 (2009)

7. M. Carpo, "Excessive Resolution: From Digital Streamlining to Computational Complexity," Archit. Des., 86, no. 6, 78-83 (2016)

8. M. J. Garcia, "A Generalized Approach to Non- Layered Fused Filament Fabrication," ACADIA 2017 Discip. Disrupt., 562-571 (2017)

9. G. Retsin and M. Jiménez García, "Discrete Computational Methods for Robotic Additive Manufacturing," Fabr. 2017, 178-184 (2017)

10. J. Sanchez and A. Andrasek, "Bloom," in Fabricate 2014, DGO-Digital original., UCL Press, 98-103 (2017)

11. A. Kaufman, D. Cohen, and R. Yagel, "Volume graphics," Computer (Long. Beach. Calif)., 26, no. 7, pp. 51-64 (1993)

12. R. Wu, H. Peng, F. Guimbretière, and S. Marschner, "Printing arbitrary meshes with a 5DOF wireframe printer," ACM Trans. Graph., 35, no. 4, pp. 1-9 (2016)

13. S. Mueller, S. Im, S. Gurevich, A. Teibrich, L. Pfisterer, F. Guimbretière, and P. Baudisch, "WirePrint: 3D Printed Previews for Fast Prototyping," in Proceedings of the 27th annual ACM symposium on User interface software and technology - UIST '14, 273280 (2014)

14. D. Reynolds, K. M. Tam, R. Otani, and E. Poulsen, "Equivalent material modelling of complex additive manufactured conformal lattices," 1-10 (2017)

15. G. Pasquarelli, W. Sharples, C. Sharples, R. Caillouet, J. Cerone, J. Gulliford, L. Mendez, J. Vereschak, J. Nardone, R. Otani, E. Poulsen, D. Reynolds, and K.-M. M. Tam, "Additive Manufacturing Revolutionizes Lightweight Gridshells," in Proceedings of the IASS Annual Symposium, (2017)

16. K. C. Cheung, "Digital Cellular Solids: Reconfigurable composite materials," Massachusetts Institute of Technology, (2012)

17. L. Gibson and M. Ashby, Cellular solids: structure and properties. Cambridge university press, (1999)

18. J. Willmann, F. Gramazio, M. Kohler, and S. Langenberg, "Digital by Material," in Rob $\mid$ Arch 2012, S. Brell-Çokcan and J. Braumann, Eds. Vienna: Springer, 12-27 (2013)

19. J. Feng, J. Fu, C. Shang, Z. Lin, and B. Li, "Porous scaffold design by solid T-splines and triply periodic minimal surfaces," Comput. Methods Appl. Mech. Eng., 336, 333-352 (2018)

20. S. Wang, L. Zhou, Z. Luo, Y. Luo, and X. Wang, "Lightweight of artificial bone models utilizing porous structures and 3D printing," Int. J. Performability Eng., 13, no. 5, 633642 (2017)

21. D. J. Yoo, "Porous scaffold design using the distance field and triply periodic minimal surface models," Biomaterials, 32, no. 31, 7741-7754 (2011)

22. L. Gibson, "The mechanical behaviour of cancellous bone," J. Biomech., 18, no. 5, 317-328 (1985)

23. M. Liebschner and M. Wettergreen, "Optimization of bone scaffold engineering for load bearing applications,” Top. Tissue Eng., Ch. 6, (2003) 
24. N. Oxman, "Virtual and Physical Prototyping Variable property rapid prototyping," 6, no. 1, 3-31 (2011)

25. N. A. Fleck, V. S. Deshpande, and M. F. Ashby, "Micro-architectured materials: Past, present and future," Proc. R. Soc. A Math. Phys. Eng. Sci., 466, no. 2121, 2495-2516 (2010)

26. D. Meagher, "Geometric modeling using octree encoding," Comput. Graph. Image Process., 19, no. 2, 129-147 (1982)

27. S. Liu, Y. Li, and N. Li, "A novel free-hanging 3D printing method for continuous carbon fiber reinforced thermoplastic lattice truss core structures," Mater. Des., 137, 235-244 (2018) 